\title{
Rituximab in Systemic Lupus Erythematosus and Lupus Nephritis
}

\author{
Hannah Beckwith Liz Lightstone \\ Imperial AHSC Lupus Centre and Section of Renal Medicine and Vascular Inflammation, Department of Medicine, \\ Imperial College London, London, UK
}

\section{Key Words}

Systemic lupus erythematosus - Lupus nephritis .

Rituximab - B cell depletion therapy

\begin{abstract}
Treatment options for systemic lupus erythematosus (SLE) and lupus nephritis (LN) have high associated morbidity and mortality. Side effects, particularly from long-term corticosteroid usage, limit patient adherence, with subsequent impacts on treatment efficacy. In addition, a subset of patients with SLE/LN fails to respond to current standard immunotherapy. There is an urgent need to develop steroid-sparing treatment regimens as well as novel therapies for the management of refractory disease. Rituximab is a chimeric mouse/human monoclonal antibody directed against the $\mathrm{B}$ cell CD20 receptor. It has been used in the treatment of nonHodgkin's lymphoma for over 30 years and has an excellent safety profile. Recent work has demonstrated a role for B cell depletion therapy in the management of autoimmune disease, and the efficacy of rituximab in many observational studies in SLE and LN has been noted. Unfortunately, two large randomised controlled trials evaluating rituximab for the treatment of renal and non-renal lupus failed to meet their primary endpoints. Reasons for this have been discussed extensively within the medical community with a general consensus that trial design (steroid use, trial size and endpoints used) was the principal reason for the failures. Despite the lack of trial evidence, clinical experience means
\end{abstract}

many physicians firmly believe in the value of rituximab in SLE/LN treatment and have continued to use it in their clinical practice. Recent work has demonstrated the efficacy of rituximab as a steroid-sparing agent and as an alternative therapeutic option for refractory SLE/LN. There are two further rituximab randomised controlled trials planned/started in $\mathrm{LN}$ - one using a steroid-minimising regimen with rituximab for induction and one evaluating rituximab for $L N$ refractory to 6 months standard of care treatment. Rituximab remains a problematic drug in lupus and $\mathrm{LN}$ - it is a biologically plausible agent with a huge amount of supportive anecdotal clinical data. Yet the completed trials have been negative to date despite clinical experience strongly suggesting efficacy. It is hoped that the two new trials will determine the role for rituximab, at least in $L N$.

c) 2014 S. Karger AG, Basel

\section{Introduction}

B cells play a pivotal role in the development and maintenance of autoimmune disease [1]. Systematic lupus erythematosus (SLE) is a prototypic autoimmune disorder associated with a diverse array of pathogenic auto-

Biologic Treatment in Glomerular Disease D. Jayne, Cambridge

V. Tesar, Prague

\section{KARGER}

E-Mail karger@karger.com

www.karger.com/nec
(C) 2014 S. Karger AG, Basel

$1660-2110 / 14 / 1284-0250 \$ 39.50 / 0$
Prof. Liz Lightstone, $\mathrm{PhD}$, FRCP

Section of Renal Medicine and Vascular Inflammation

Department of Medicine, Imperial College London

Hammersmith Campus, Du Cane Road, London W12 0NN (UK)

E-Mail l.lightstone@imperial.ac.uk 
antibodies directed towards cytoplasmic and nuclear cell compartments. Multiple systems can be involved, with subsequent immune complex deposition driving complement activation, inflammation and end-organ damage. Between 40 and $70 \%$ of patients with SLE develop lupus nephritis (LN), and the consequences can be devastating [2]. Even with aggressive immunosuppressive therapy, rates of end-stage renal disease over 5-10 years are in the region of $10 \%$, a figure that has disappointingly remained largely unchanged over the last 30 years $[3,4]$. Despite the introduction of newer immunosuppressive agents such as mycophenolate mofetil (MMF), treatment of SLE and LN remains challenging. Current standard therapeutic regimens are associated with significant side effects and cumulative toxicity including premature ovarian failure, increased risk of future malignancies and teratogenicity. Prolonged corticosteroid use is associated with increased risk of premature cardiovascular disease and a 2- to 4-fold increased risk of coronary events in SLE [5]. In addition, cosmetic changes associated with prolonged steroid use may contribute to non-adherence, which is a recognised cause of treatment failure [6]. Consequently, there has been a strong desire amongst physicians to develop new and innovative treatment regimens to help improve prognosis and reduce the burden of iatrogenic morbidity [7]. Given the key role of B cells in the development of a dysregulated immune response, a strong argument exists for the use of B cell-depleting agents in the management of SLE and LN.

\section{Rituximab}

Rituximab is a chimeric mouse/human monoclonal antibody directed against the CD20 receptor of the $\mathrm{B}$ cell. CD20 is expressed on immature, mature and activated B cells, but not plasma cells, stem cells or pro-B cells. Rituximab acts via antibody-dependent cell-mediated cytotoxicity, complement-dependent cytotoxicity and apoptosis to effectively deplete B cells for 6-9 months in over $80 \%$ of patients [8]. Importantly, rituximab has been extensively used in the treatment of non-Hodgkin's lymphoma [9] and has an excellent safety record. Adverse events following rituximab use are minimal and include infusionrelated reactions and increased risk of infection $[7,10]$. Hence, there was confidence in transferring its use to autoimmune diseases, and rituximab has been successfully used in the treatment of rheumatoid arthritis, ANCA-associated systemic vasculitis and primary immune thrombocytopenic purpura [11-13].

Rituximab in SLE and LN

\section{Rituximab in SLE}

SLE is an archetypal antibody-mediated disease and hence promoting $B$ cell depletion appears to target a plausible critical pathophysiological pathway. Early case reports and then case series suggested benefit in the treatment of SLE (reviewed in [14]). This led to the development of the EXPLORER trial (a randomised, double-blind, placebo-controlled exploratory phase II/III SLE evaluation of rituximab) [15]. The EXPLORER trial recruited 257 patients aged 16-75 years with moderate and severe SLE who met four of the American College of Rheumatology (ACR) criteria for SLE including a positive test for antinuclear antibodies, active disease at screening [defined as $\geq 1$ domain with a British Isles Lupus Assessment Group (BILAG) A score (severe activity) or $\geq 2$ domains with a BILAG B score (moderate disease activity)], and stable use of one immunosuppressive drug at entry which was continued throughout the trial (methotrexate, azathioprine or MMF). 57\% of participants were corticosteroid dependent. Exclusion criteria included severe central nervous system or organ-threatening lupus, any other active conditions requiring significant use of immunosuppressives, previous B cell depletion therapy, and deranged hepatic or haematological serum parameters. Patients were randomised at a 2:1 ratio to receive intravenous rituximab or placebo on days 1, 15, 168 and 182 (in addition to prednisolone and baseline immunosuppressive therapy). The primary endpoint was the effect of placebo versus rituximab in achieving and maintaining clinical response at week 52, assessed using the BILAG index organ system scores.

Unfortunately the EXPLORER trial failed to meet its primary endpoint. Potential reasons for this include inadequate trial size, concomitant background immunosuppressive/high-dose corticosteroid usage and apportion of endpoints which were unable to discriminate between minor disease fluctuations and meaningful clinical response [16]. However, on further examination, a post hoc analysis of this trial suggested rituximab reduced the risk of a subsequent first severe flare and lowered mean severe flare rates [17]. In addition, efficacy signals were detected in subsets of patients including African Americans and Hispanics [15].

It is worth briefly reviewing the contrasting fate of the anti-BLys antibody, belimumab. B lymphocyte-stimulating factor (BLyS) is a cytokine produced predominantly by myeloid cells. BLyS is necessary for B cell maturation, survival and immunoglobulin production [16]. Belumimab, a soluble anti-BLyS monoclonal antibody was 
shown to lead to superior results when added to standard of care, compared to standard of care alone, in two large randomised controlled trials in patients with active SLE $(\mathrm{n}=865$ and 819) $[18,19]$. These results led to belimumab being granted regulatory approval for the treatment of patients with antibody-positive SLE with active disease despite treatment with standard drugs.

These trials were designed slightly differently to the EXPLORER trial; they used large numbers of patients and aimed for a smaller delta. A composite endpoint was used, the SLE Responder Index (SRI) response rate at week 52 [which is largely driven by the SLE Disease Activity Index (SLEDAI) score], and inclusion criteria were narrower - only seropositive patients were included and they had to have evidence of activity as judged by a SLEDAI score of at least 6. Key exclusions were central nervous system lupus or active LN. Participants were again continued on previous immunosuppressive therapy, but adjustments to background immunosuppressants were more strictly controlled and time limited, including increases to corticosteroid doses in the first 6 months and increases to other immunosuppressants in the first 16 weeks.

Interestingly, a post hoc analysis of the belimumab studies demonstrated that belimumab is most effective in the subset of patients with high disease activity (e.g. high titres of anti-dsDNA antibodies and low complement levels) [20]. Rituximab also reduces anti-dsDNA levels and increased complement levels $[15,21]$, suggesting that the problem might not be the drug, but instead the trial design.

\section{Rituximab in LN}

Given the many case series and registry reports of use in refractory $\mathrm{LN}$, it was reasonable to evaluate rituximab in a clinical trial setting for the treatment of LN. LN is classified according to the ISN/RPS criteria [22]. Treatment is generally reserved for those with proliferative LN (class III-a or a/c or class IV-S a or a/c or class IV-G a or $\mathrm{a} / \mathrm{c}$ where $\mathrm{a}=$ active, $\mathrm{c}=$ chronic, $\mathrm{S}=$ segmental and $\mathrm{G}=$ Global - all referring to glomerular lesions), and some guidelines recommend treatment for class V LN only if associated with nephrotic range proteinuria [23].

Following the failure of the EXPLORER trial (non-renal SLE) to meet its primary endpoint of reduced clinical activity without subsequent flares [15], guidelines for steroid use in the LUNAR trial (Lupus Nephritis Assessment with Rituximab) were more stringent. However, ritux- imab was again used as an add-on to standard of care with MMF and corticosteroids. Unfortunately, the LUNAR trial also failed to meet its primary endpoint of $20 \%$ superiority in the proportion of patients achieving complete or partial remission at 52 weeks [21].

Trial design is again likely to have contributed to its lack of success. The use of corticosteroids as well as MMF as background in both the rituximab and control group may have masked any incremental benefit of rituximab. In addition, the LUNAR trial was powered for a composite endpoint of complete renal remission (CR) and partial renal remission (PR), but weighted towards a greater proportion of CR rather than PR (powered at $90 \%$ to detect a $20 \%$ increase in CR and a $5 \%$ increase in PR). This meant the study was underpowered to detect the $15 \%$ increase in the proportion of PR actually seen or doubling of the number of rituximab-treated patients who achieved a PR compared to standard therapy (31 vs. $15 \%$ ). Ironically, the overall delta was $11 \%$, which was larger than that seen in the belimumab trials but in a much smaller trial so the difference was not significant. However, there are reasons to consider that the rituximab arm showed benefit. The data at 78 weeks of follow-up strongly suggest that rituximab was having a beneficial effect as there were significant differences in the proportion of patients who achieved proteinuric remission and who did not require additional immunosuppression (reviewed in [24]). Repeat renal biopsies, had they been done, might have demonstrated histological remission at 1 year which we have suggested may precede biochemical remission which can take a long time as suggested by these data. What is clinically important is that despite the failure of these two rituximab randomised controlled trials to achieve their primary endpoint, many clinicians continue to use rituximab in daily practice, based on their personal experience, the large case series and registry data, and the long-term outcome data in the LUNAR trial $[1,14,25]$. This contrasts with the clinical reality for belimumab, where despite the positive trial data there has been relatively slow uptake of use of this drug in lupus, possibly because of difficulty in knowing which patients would benefit most and due to the lack of data on use in LN.

We have developed an alternative way of using rituximab - we chose to use rituximab as an instead of drug rather than an add-on and (more radically) opted to omit oral steroids. The rationale was based on experience in renal transplantation where the introduction of biological agents at induction has led to the development of steroid-avoiding regimens. Importantly, in transplantation such an approach has led to better outcomes both in 
terms of lower rates of rejection and of fewer side effects such as new-onset diabetes [26]. We have reported our single-centre observational cohort study of 50 consecutive patients with biopsy-proven LN, not on oral steroids and who were treated with our 'Rituxilup' regimen: methyl prednisolone $(500 \mathrm{mg}$ ) and rituximab (1 g) on days 1 and 15 and with just MMF as maintenance, and no oral steroids [27]. The results were very encouraging - 52\% of patients achieved complete renal remission at 1 year, there were few extrarenal flares and of the 45 patients who stayed on the regimen, only 2 required longer than 2 weeks of oral steroids over a median follow-up of 163 weeks. Colleagues have used this approach in new patients with lupus (not specifically renal lupus) and have also demonstrated efficacy and steroid sparing [28]. There has been great excitement in the renal and rheumatology communities over a steroid-sparing approach. This work is now being taken forward in a large, openlabelled, randomized, controlled, multicentre trial (the RITUXILUP trial, NCT01773636 at www.clinicaltrials. gov). The trial is designed as a non-inferiority trial to demonstrate that the Rituxilup regimen (Rituximab, methyl prednisolone and MMF, but no oral steroids) is not inferior in inducing CR at 1 year compared to standard of care with methyl prednisolone, MMF and tapering oral steroids. It is also powered to show superiority of the Rituxilup regimen compared to standard of care for serious adverse events and serious infections. Patients $(\mathrm{n}=252)$ will be followed for a minimum of 2 years. The estimated completion date is 2018 , and if the trial is a success it will mark a paradigm shift in the management of LN as it will be the first time in 66 years that steroids are not deemed necessary for treatment.

RING (Rituximab for Lupus Nephritis with Remission as a Goal, NCT01673295), another ongoing trial led by
Houssiau and colleagues, is aiming to evaluate the addition of rituximab in patients who have had 6 months of standard of care but failed to achieve a complete remission. This trial will formally assess whether rituximab is effective in refractory $\mathrm{LN}$.

So, several years after the first reports of rituximab as the new 'wonder' drug for lupus and LN, it remains unlicensed but quite widely used - in that respect it is no different from MMF, cyclophosphamide or azathioprine. Guidelines on the management of LN suggest it can be used (added in) where standard of care with MMF or cyclophoshphamide has failed in the treatment of proliferative LN [29-31].

\section{Conclusion}

Whilst no randomised controlled trial presently exists demonstrating the efficacy of rituximab in treating SLE and LN, it is clear that clinicians and scientists have a strong belief in its potential role based on biological plausibility (the key role of B cells in lupus pathogenesis), the influence on serology and surrogate markers of disease activity, and numerous cohort and registry reports. Part of the drive to use rituximab is that it has a strong and long safety profile and that current treatment options have a high associated morbidity and mortality, and side effects experienced by patients limit adherence and treatment efficacy. There is an unmet need for both steroid-sparing treatment regimens and new novel therapies to treat refractory cases: rituximab is an exciting therapeutic option which has demonstrated success in both of the above. Further work is needed to elucidate the real value of $B$ cell depletion through rituximab.

\section{References}

$>1$ Tobon GJ, Izquierdo JH, Canas CA: B lymphocytes: development, tolerance, and their role in autoimmunity - focus on systemic lupus erythematosus. Autoimmune Dis 2013, DOI: $10.1155 / 2013 / 827254$.

-2 Pollak VE, Pirani CL, Schwartz FD: The natural history of the renal manifestations of systemic lupus erythematosus. J Lab Clin Med 1964;63:537-550.

-3 Croca SC, Rodrigues T, Isenberg DA: Assessment of a lupus nephritis cohort over a 30year period. Rheumatology (Oxford) 2011;50: 1424-1430.

Rituximab in SLE and LN
4 Ward MM: Changes in the incidence of endstage renal disease due to lupus nephritis in the United States, 1996-2004. J Rheumatol 2009;36:63-67.

5 Nikpour M, et al: Importance of cumulative exposure to elevated cholesterol and blood pressure in development of atherosclerotic coronary artery disease in systemic lupus erythematosus: a prospective proof-of-concept cohort study. Arthritis Res Ther 2011;13:R156.

6 Masood S, Jayne D, Karim Y: Beyond immunosuppression - challenges in the clinical management of lupus nephritis. Lupus 2009; 18:106-115.
7 Pepper R, et al: Rituximab is an effective treatment for lupus nephritis and allows a reduction in maintenance steroids. Nephrol Dial Transplant 2009;24:3717-3723.

8 Anolik JH, et al: The relationship of FcgammaRIIIa genotype to degree of $B$ cell depletion by rituximab in the treatment of systemic lupus erythematosus. Arthritis Rheum 2003;48: 455-459.

9 Plosker GL, Figgitt DP: Rituximab: a review of its use in non-Hodgkin's lymphoma and chronic lymphocytic leukaemia. Drugs 2003; 63:803-843. 
10 Turner-Stokes T, et al: The efficacy of repeated treatment with B-cell depletion therapy in systemic lupus erythematosus: an evaluation. Rheumatology (Oxford) 2011;50:1401-1408.

11 Edwards JC, et al: Efficacy of B-cell-targeted therapy with rituximab in patients with rheumatoid arthritis. N Engl J Med 2004;350: 2572-2581.

12 Ferraro AJ, et al: Effective therapeutic use of rituximab in refractory Wegener's granulomatosis. Nephrol Dial Transplant 2005;20: 622-625.

13 Patel VL, et al: Outcomes 5 years after response to rituximab therapy in children and adults with immune thrombocytopenia. Blood 2012;119:5989-5995.

14 Favas C, Isenberg DA: B-cell-depletion therapy in SLE - what are the current prospects for its acceptance? Nat Rev Rheumatol 2009;5: 711-716.

15 Merrill JT, et al: Efficacy and safety of rituximab in moderately-to-severely active systemic lupus erythematosus: the randomized, double-blind, phase II/III systemic lupus erythematosus evaluation of rituximab trial. Arthritis Rheum 2010;62:222-233.

16 Thanou A, Merrill JT: Treatment of systemic lupus erythematosus: new therapeutic avenues and blind alleys. Nat Rev Rheumatol 2014;10:23-34.
17 Merrill J, et al: Assessment of flares in lupus patients enrolled in a phase II/III study of rituximab (EXPLORER). Lupus 2011;20:709716.

18 Furie R, et al: A phase III, randomized, placebo-controlled study of belimumab, a monoclonal antibody that inhibits B lymphocyte stimulator, in patients with systemic lupus erythematosus. Arthritis Rheum 2011;63: 3918-3930.

19 Navarra SV, et al: Efficacy and safety of belimumab in patients with active systemic lupus erythematosus: a randomised, placebo-controlled, phase 3 trial. Lancet 2011;377:721731.

20 van Vollenhoven RF, et al: Belimumab in the treatment of systemic lupus erythematosus: high disease activity predictors of response. Ann Rheum Dis 2012;71:1343-1349.

21 Rovin BH, et al: Efficacy and safety of rituximab in patients with active proliferative lupus nephritis: the Lupus Nephritis Assessment with Rituximab study. Arthritis Rheum 2012;64:1215-1226.

22 Weening JJ, et al: The classification of glomerulonephritis in systemic lupus erythematosus revisited. J Am Soc Nephrol 2004;15:241-250.

23 Radhakrishnan J, Cattran DC: The KDIGO practice guideline on glomerulonephritis: reading between the (guide)lines - application to the individual patient. Kidney Int 2012;82:840-856.

24 Lightstone L: The landscape after LUNAR: rituximab's crater-filled path. Arthritis Rheum 2012;64:962-965.
25 Gunnarsson I, Jonsdottir T: Rituximab treatment in lupus nephritis - where do we stand? Lupus 2013;22:381-389.

26 Lightstone L: Minimising steroids in lupus nephritis - will B cell depletion pave the way? Lupus 2013;22:390-399.

27 Condon MB, et al: Prospective observational single-centre cohort study to evaluate the effectiveness of treating lupus nephritis with rituximab and mycophenolate mofetil but no oral steroids. Ann Rheum Dis 2013;72:12801286.

28 Ezeonyeji AN, Isenberg DA: Early treatment with rituximab in newly diagnosed systemic lupus erythematosus patients: a steroid-sparing regimen. Rheumatology (Oxford) 2012; 51:476-481.

29 Bertsias GK, et al: Joint European League against Rheumatism and European Renal Association-European Dialysis and Transplant Association (EULAR/ERA-EDTA) recommendations for the management of adult and paediatric lupus nephritis. Ann Rheum Dis 2012;71:1771-1782.

30 Hahn BH, et al: American College of Rheumatology guidelines for screening, treatment, and management of lupus nephritis. Arthritis Care Res (Hoboken) 2012;64:797-808.

31 Kidney Disease: Improving Global Outcomes (KDIGO) Glomerulonephritis Working Group. Kidney Int (Suppl) 2012;2:221232 